\title{
Erratum
}

\section{Corrections to "Multitarget Identification and Localization Using Bistatic MIMO Radar Systems"}

\author{
Haidong Yan, Jun Li, and Guisheng Liao \\ National Lab of Radar Signal Processing, Xidian University, Xi'an 710071, China \\ Correspondence should be addressed to Jun Li, junli01@mail.xidian.edu.cn \\ Received 29 February 2008; Accepted 29 February 2008
}

Copyright (C) 2008 Haidong Yan et al. This is an open access article distributed under the Creative Commons Attribution License, which permits unrestricted use, distribution, and reproduction in any medium, provided the original work is properly cited.

Firstly, the second term $\mathbf{v}$ of (4) in [1] is zero-mean complex Gaussian with $\mathbf{v} \sim N^{c}\left(0, \sigma_{v}^{2} \mathbf{I}_{N M}\right)$, where $\sigma_{v}=(1 / \sqrt{L}) \sigma_{w}$. Also, the $\sigma_{w}^{2}$ in (8) should be changed to $\sigma_{\mathbf{v}}^{2}$. Secondly, if we have $Q$-independent pulses, the Fisher information matrix in (9) should be [2]

$$
\mathbf{J}(\boldsymbol{\xi})=Q \operatorname{tr}\left[\mathbf{R}_{\eta}^{-1}(\xi) \frac{\partial \mathbf{R}_{\eta}(\xi)}{\partial \xi} \mathbf{R}_{\eta}^{-1}(\xi) \frac{\partial \mathbf{R}_{\eta}(\xi)}{\partial \xi}\right] .
$$

The corresponding formulas of CRB in the appendix should be revised.

Owing to these corrections, Figures 3, 4, 5, and 6 of the original submission would require small adjustments.

\section{REFERENCES}

[1] H. Yan, J. Li, and G. Liao, "Multitarget identification and localization using bistatic MIMO radar systems," EURASIP Journal on Advances in Signal Processing, vol. 2008, Article ID 283483, 8 pages, 2008.

[2] H. L. van Trees, Optimum Array Processing: Part IV, John Wiley \& Sons, New York, NY, USA, 2002. 